\title{
Die Liste als Ordnungsmedium in mittelalterlichen Libri vitae
}

\author{
Michelle Waldispühl
}

Online publiziert: 16. April 2019

(C) Der/die Autor(en) 2019

Zusammenfassung Listen bilden ein historisches und kulturelles Kontinuum in der Schriftverwendung und stellen auch in mittelalterlichen Libri vitae das grundsätzliche Ordnungsmedium dar. Die Bücher wurden im späten 8. und frühen 9. Jahrhundert in mehreren Klöstern als Verbrüderungs- und Gedenkbücher angelegt und enthalten umfangreiche Listen von Personennamen. Während diese Quellen und ihre Namenlisten bisher hauptsächlich Gegenstand von historischen und namenkundlichen Studien waren, diskutiert dieser Beitrag die Textsorte $>$ Liste $<$ in den Codices in Bezug auf ihre formalen, inhaltlichen und funktionalen Eigenschaften. Es wird eine große Vielfalt an formalen und inhaltlichen Prinzipien in den Listen der Libri vitae festgestellt, was einerseits mit funktionalen Verschiebungen im Gebrauch der Manuskripte im Laufe der Zeit und andererseits mit verschiedenen institutionellen und politischen Kontexten sowie idiosynkratischen Schreibpraktiken in Verbindung gebracht werden kann. Diese flexible Handhabung unterstreicht das enorm breite funktionale Potential der Liste als Kulturpraxis und als Medium der sozialen Ordnung. Es zeigen sich jedoch auch semiotische Konstanten über alle Listen hinweg, die als Ausdruck von etablierten mittelalterlichen Schreibpraktiken angesehen werden können.

Schlüsselwörter Libri vitae $\cdot$ Verbrüderungsbücher $\cdot$ mittelalterliche

Personennamen $\cdot$ Liste $\cdot$ historische Textlinguistik $\cdot$ mittelalterliche

Schreibpraktiken

M. Waldispühl ( $ه)$

Institutionen för språk och litteraturer, Göteborgs universitet, Box 200, 40530 Göteborg, Schweden

E-Mail: michelle.waldispuhl@sprak.gu.se 


\section{The List as a Medium of Arrangement in Medieval Libri vitae}

Abstract Lists form a historical and cultural continuum in the use of writing and also constitute the basic principle of arrangement in medieval Libri vitae. These books consist of extensive lists of personal names and became popular in Carolingian late eighth and early ninth century in several medieval monasteries. They had memorial function for the person named in the lists. Whereas these books and their name lists have been mainly subject to historical and onomastic research so far, this article aims at approaching the nature of the text type >list< present in the codices on a formal, textual and functional level. The analysis shows a wide variety of formal and textual principles underlying the presentation of lists in Libri vitae which can be related to functional shifts in the use of the manuscripts in the course of time on the one hand and on different institutional and political contexts as well as idiosyncratic scribal practices on the other. This flexible handling underlines the enormously broad functional potential of the list as a cultural practice and as a medium of social arrangement. However, there are also semiotic constants in all of the lists manifesting established medieval writing practices.

Keywords Libri vitae $\cdot$ Fraternity Books $\cdot$ Medieval Personal Names $\cdot$ Historical Text Linguistics $\cdot$ List $\cdot$ Medieval Writing Practices

\section{Einführendes}

»The list offers a marvelous venue for literary and linguistic spectacle « (Belknap 2004, S. 35), und die Faszination für diese Textsorte hat eine lange Tradition. Listen stehen am Anfang der phylogenetischen Schriftlichkeitsgeschichte und bilden ein historisches und kulturelles Kontinuum in der Schriftverwendung. Aus allen Epochen und Schriftkulturen lassen sich Beispiele von praktischen Listen finden, in denen das ordnende Potential des schriftlichen Mediums ausgenutzt wurde die Beispiele reichen von administrativen Listen städtischer Transaktionen im alten Mesopotamien über elaborierte Formen von Systematisierungen enzyklopädischen Wissens in der Frühen Neuzeit und alltäglichen Einkaufslisten (vgl. Goody 1977, S. 82-103) bis zu Bulletpoint-Listen in Powerpoint und Google-Trefferlisten (vgl. Ledin 2015, S. 19). Neben die praktischen Listen treten die ästhetisierten Listen in der Literatur. Homers Aufzählung der kriegerischen Flotten, beispielsweise, poetisiert eine schwindelerregende Anzahl und die Aura des Unendlichen, während Rabelais' Auflistung der Praktiken des Arschwischens die Praxis des extensiven Katalogisierens in der Frühen Neuzeit poetisch ironisiert (vgl. Eco 2009, S. 17 f., 249).

Während wir sowohl in der Alltagsschriftlichkeit als auch in allen möglichen anderen schriftlichen Domänen von Listen umgeben sind, hat diese Textsorte in der Forschung bisher nur spärlich Beachtung gefunden. Als eine der ausführlichsten Betrachtungen gilt Jack Goodys (1977, S. 74-111) anthropologische Analyse der Liste als zentrales Phänomen der Schriftlichkeitsgeschichte. Er sieht die Liste als wichtiges Instrument in der Entwicklung der menschlichen Kognition an, was insgesamt im Kontext seiner Forschung zur Kulturtechnik Schrift und seiner evolutionären Vor- 
stellung von Schriftkulturen in Abgrenzung zu oralen Gesellschaften einzuordnen ist. Weitere zentrale Beiträge, die insbesondere auch theoretische Ansätze thematisieren und systematisieren, stammen bisher vorwiegend aus der Literaturwissenschaft (vgl. Abschnitt 3), sprachwissenschaftliche und interdisziplinäre Betrachtungen zur Liste begrenzen sich auf ein paar wenige Einzelstudien und Sammelbände (Doležalová 2009a; Leonardi et al. 1995; Ledin 2015).

Dieser Beitrag widmet sich den Listen einer bestimmten mittelalterlichen Textform - den Libri vitae, beziehungsweise Verbrüderungsbüchern, bestehend aus extensiven Listen von Personennamen, die hauptsächlich zum Zweck des Gebetsgedenkens verzeichnet wurden (vgl. Abschnitt 2). Dieser Quellentyp stand bisher vorwiegend im Interesse der Geschichtswissenschaften, welche die Listen prosopographischen Studien sowie Untersuchungen von sozialer Zusammengehörigkeit der in den Listen genannten Personen und mittelalterlichen gesellschaftlicher Ordnungen zu Grunde legten. ${ }^{1}$ Daneben gibt es vereinzelte namenkundliche Studien insbesondere zu Nameninventaren, -etymologien und historischen Benennungspraktiken (z.B. Wagner 2011; Insley 2015). In diesem Artikel sollen die Namenlisten in den Libri vitae aus interdisziplinärer Perspektive beleuchtet werden und zwar anhand von Gesichtspunkten, die sich aus der Liste als Textsorte ableiten lassen. Dabei entwerfe ich ein analytisches Modell, das Ansätze der bisherigen Listen-Forschung, die formale Eigenschaften der Liste wie auch kulturanthropologische und sozialsemiotische Deutungsmuster tangieren, aufgreift (vgl. Abschnitt 3).

Das Ziel des Beitrags ist ein zweifaches. Zum einen hat der Beitrag für die Erforschung der mittelalterlichen Libri vitae explorativen Charakter und es sollen Möglichkeiten eines analytischen Mehrwerts dieses Zugangs exemplarisch aufgezeigt werden. Es besteht somit nicht der Anspruch, eine erschöpfende Darstellung der Listen in Libri vitae zu bieten - dies wäre Gegenstand einer größer angelegten Studie. Zum anderen systematisiert dieser Beitrag für die Listen-Forschung bisherige theoretische Ansätze zur Textsorte und erweitert sie um Aspekte aus den überlieferten Listen in Libri vitae.

\section{Libri vitae}

Der Brauch der Libri vitae (auch Verbrüderungsbücher, Gedenkbücher, Libri memoriales) geht auf die biblische Vorstellung eines Himmlischen Buchs des Lebens zurück, in dem diejenigen Personen mit Namen eingeschrieben sind, die am Jüngsten Tag in Gottes Reich eingelassen werden (Offb. 3,5; 13,8; 20,12-15). Mit der Eintragung seines Namens in eine Liber vitae-Handschrift eines Konvents »verband sich für den Gläubigen die Hoffnung, durch Gebet und Fürbitte Aufnahme in jenes Buch des Lebens zu finden, das der Herr am Jüngsten Tag öffnen würde « (Geuenich/ Ludwig 2015b, S. 9). Es stand somit im persönlichen Interesse der Gläubigen, ihre Namen in einem Liber vitae festzuhalten.

\footnotetext{
1 Vgl. zentrale Beiträge in Ludwig (2008) und jüngst auch Geuenich/Ludwig (2015a).
} 
Die ersten Libri vitae wurden Ende des 8. Jahrhunderts und im frühen 9. Jahrhundert in den Klöstern von Salzburg, ${ }^{2}$ Reichenau, ${ }^{3}$ St. Gallen, ${ }^{4}$ Pfäfers ${ }^{5}$ und Remiremont $^{6}$ planmäßig angelegt (vgl. die Übersicht bei Geuenich 2015, S. 125 f.) und waren laut Geuenich/Ludwig (2015b, S. 9f.) formal noch stark an den Diptychen orientiert. Die Handschriften hatten in der Zeit ihrer Anlage jedoch nicht nur memoriale und liturgische Funktion, indem die Nameneintragungen im Gottesdienst verwendet wurden (Geuenich/Ludwig 2015b: 11) und eventuell in Teilen gar verlesen wurden, sondern gaben gesellschaftliche Ordnungen und soziale Bindungen wieder, die auch auf politischen Absichten fußten. Im Anschluss an den Gebetsbund von Attigny im Jahr 762 verpflichteten sich verschiedene Konvente des Frankenreiches zum gegenseitigen Gebetsgedenken, was durch den Austausch von Namenlisten ihrer Angehörigen gewährleistet wurde (vgl. Schmid/Oexle 1974, Lieven 2015, S. 248f.). Nicht alle Libri vitae wurden als separate Handschriften angelegt - einige Namenlisten wurden in Evangeliaren ergänzt und erscheinen neben biblischen und liturgischen Texten. Dies ist beim Liber Viventium Fabariensis aus Pfäfers der Fall wie auch bei den später im 9. und im 10. Jahrhundert entstandenen Memorialeinträgen von San Salvatore / Santa Lucia in Brescia ${ }^{7}$ und jenen von Cividale ${ }^{8}$ (Geuenich 2015, S. 126). Ebenso wenig stellen Libri vitae ein ausschließlich kontinentales Phänomen dar. Sie waren auch in England populär, wo drei Exemplare überliefert sind (Durham Liber vitae, ${ }^{9}$ 9. Jahrhundert; Liber vitae of Thorney Abbey ${ }^{10}$ und Liber vitae of New Minster and Hyde Abbey, ${ }^{11} 11$. Jahrhundert).

Ein wichtiges Charakteristikum der Namenlisten in Libri vitae ist ihr teilweise immenser Umfang und ihre Dynamik. Sie wurden laufend und mehr oder weniger systematisch ergänzt und bearbeitet. Das Verbrüderungsbuch von Reichenau stellt den umfangreichsten überlieferten Liber vitae dar mit mehr als 38.000 Nameneinträgen. Die Handschrift wurde im ersten Drittel des 9. Jahrhunderts angelegt und bis ins 13. Jahrhundert kamen Namen hinzu - der Codex war somit über 400 Jahre lang in Verwendung.

\footnotetext{
2 Stiftsarchiv St. Peter, Salzburg. Faksimile: Forstner (1974).

3 Zentralbibliothek Zürich, Ms. Rh. hist. 27. Faksimile und Edition: Autenrieth, Geuenich/Schmid (1979). Die digitalisierte Handschrift kann auf www.e-codices.ch eingesehen werden. Zur Illustration der folgenden Ausführungen wird auf diese Internetseite verwiesen.

4 Stiftsarchiv St. Gallen, Class. I. Cist. C3. B 55. Edition: Geuenich/Ludwig, in Vorbereitung.

5 Stiftsarchiv St. Gallen, Fonds Pfäfers Codex 1. Faksimile-Edition: Bruckner, Sennhauser/Perret (1973).

${ }^{6}$ Biblioteca Angelica, Rom, Ms. 10. Edition: Hlawitschka/Schmid/Tellenbach (1970).

7 Biblioteca Queriniana, Brescia, Cod. G VI. 7. Edition: Geuenich/Ludwig (2000).

8 Museo Archeologico Nazionale, Cividale, Codex XXXXVIII. Vgl. Ludwig (1999: 245-277).

9 London, British Library MS Cotton Domitian A. VII. Edition: Rollason/Rollason (2007).

10 London, British Library Additional MS. 40, 000, fols. 1v, 2r, 2v, 3r, 3v, 4r, 9v, 10r, 10v, 11r, 11v, 12r. Edition: Rollason (2015).

11 London, British Library Stowe 944. Faksimile: Keynes (1996).
} 


\section{Analysemodell: Was ist eine Liste?}

Obwohl in der Forschung grundsätzlich im Rahmen der Möglichkeiten des schriftlichen Mediums diskutiert, beschränkt sich die Liste nicht auf das Schriftliche, sondern kommt auch in der Mündlichkeit mit ihren spezifischen Charakteristika vor (vgl. Karlsson 2010). Eco (2009) fasst den Listenbegriff gar noch weiter und bezieht auch reihende Darstellungen in der bildlichen Kunst mit ein. Mit Blick auf die Listen in Libri vitae beschränke ich mich in diesem Beitrag jedoch auf geschriebene Listen einerseits und auf praktische Listen andererseits. Aus der literaturwissenschaftlichen Forschung zu ästhetisierten Listen ziehe ich allerdings allgemein theoretische Gesichtspunkte zur Textsorte bei.

Als Ausgangspunkt der Betrachtungen dient die Definition bei Belknap (2004, S. 2): »At their most simple, lists are frameworks that hold separate and disparate items together. Lists are plastic, flexible structures in which an array of constituent units coheres through specific relations generated by specific forces of attraction.« In dieser Definition sehe ich folgende grundlegende Aspekte:

1) Listen bilden einen formalen Rahmen, der einzelne Elemente zu einem Ganzen verbindet;

2) Listen sind materiell fixiert;

3) Listen sind flexible Strukturen, d.h. in ihren inneren formalen Eigenschaften nicht prädisponiert;

4) Die einzelnen Elemente in einer Liste sind kohärent, es besteht eine innere inhaltliche Ordnung;

5) Die Ordnung wird generiert, d.h. ist nach einem bestimmten Zweck gerichtet.

Aus diesen fünf Punkten lassen sich drei übergreifende Analysekategorien ableiten, die ich im Folgenden noch weiter konkretisieren möchte: a) Form (1-3), b) Kohärenz (4), c) pragmatische Funktion (5).

a) Form Eva von Contzen (2016, S. 243, 245), die für ihre literaturhistorische Studie der Liste ebenso von obengenannter Definition ausgeht, betont die Wichtigkeit der Form für die Analyse der Liste und vereinfacht Belknaps Definition gar zu »a list is a set of items assembled in a formal unit«. Im Zusammenhang der formalen Eigenschaften ist weiter die zentrale Rolle der Materialität einer Liste in ihrer schriftlichen Fixierung zu erwähnen, worunter sowohl die Art des Schriftträgers und der Materialtyp der Schreibgrundlage als auch die visuelle Darstellung und die Nutzung beziehungsweise Gestaltung des Schriftraums fallen. Beispielsweise können einzelne Elemente in der Vertikale, d.h. in Kolumnen, oder in der Horizontale z. B. durch Komma, Punkt oder Zwischenraum getrennt - aneinandergereiht sein. Der Platz für die Aneinanderreihung kann durch Hilfs- oder Rahmenlinien markiert sein oder nicht, außerdem ist er gewöhnlich durch die Eigenschaften des Schriftträgers natürlich begrenzt. $\mathrm{Zu}$ den formalen Eigenschaften gehören zudem weitere Arten der Schriftraumgestaltung wie Unterstreichung, Abstände und dergleichen sowie Möglichkeiten der Schriftgestaltung wie etwa Schrifttyp, Kursivierung, Majuskelschreibung. 
b) Kohärenz und semantische Ordnung Die Liste als Gesamtes stiftet Kohärenz, indem einzelne Elemente nach einem bestimmten Kriterium klassifiziert werden. Die Auswahl von Elementen für eine Liste an sich schafft schon eine semantische Klassifikation, da sie gewisse Elemente integriert, andere jedoch ausschließt (vgl. Goody 1977, S. 103). Ledin (2015, S. 20) spricht in seinem semiotischen Modell der Liste und der Tabelle von »paradigmatischer Kategorisierung «, im Anschluss an Barthes' Paradigmen-Begriff, d.h. einzelne Elemente werden einem gemeinsamen Phänomen oder einer übergreifenden kulturellen Praxis zugeordnet. ${ }^{12}$ Diese Kategorisierung kann in Listen auch durch die Nennung eines Hyperonyms z. B. in einer Überschrift »Vögel Europas « sprachlich ausgedrückt sein, wie es in Glossaren und Enzyklopädien üblicherweise der Fall ist. Listen haben somit ein großes Potential als »sprachlich und medial konstruierte Formen [...], die soziale und epistemische Ordnungen konstituieren« (Schaffrick/Werber 2017, S. 304). Die Liste verbindet diskontinuierliche, einzelne Elemente in einem gemeinsamen Listenraum zu einem Kollektiv (vgl. Stäheli 2012, 93, 96). Für historische Textdokumente macht Doležalová (2009c, S. 98 f.) in diesem Zusammenhang allerdings auf die methodische Gefahr aufmerksam, dass die grundsätzliche Annahme einer Listenkohärenz dazu führen kann, dass sie hineininterpretiert wird, wenn sie im Dokument selbst nicht offenbar wird und damit nicht zwingend die zeitgenössisch historische Kohärenz erfasst, sondern anachronistische Kategorien aufgesetzt werden.

Unabhängig von der Form der Liste kann die Auflistung einzelner Elemente zu einer gewissen Kategorie entweder ausgeschöpft (geschlossen) oder offen sein. Inventarlisten aller Gegenstände eines Museums stellen beispielsweise geschlossene Listen dar, wohingegen das Museumsgästebuch offen ist und weitere Einträge laufend hinzukommen. Die einzelnen Elemente in praktischen Listen sind oft nominal und beziehen sich referentiell auf außerweltliche Dinge (Goody 1977, S. 81, 85; Belknap, S. 19): die Einträge auf einer Einkaufsliste beispielsweise findet man im Geschäft, die Personen auf der Gästeliste kommen zum Fest.

Schließlich kann die Reihung der Elemente innerhalb der Liste einer inneren semantischen Ordnung folgen und nach einem bestimmten Prinzip geschehen, z. B. in zeitlicher Abfolge, alphabetisch, nach Wichtigkeit, nach Salienz, nach logischen Parametern (vom Generellen zum Spezifischen) oder gemäß kulturellen Codes (Abfolge der Räume in einer Wohnung nach dem, wie man sie durchschreiten würde) (Fludernik 2016, S. 209f.). Listen können aber auch ungeordnet sein (Elemente in der Reihenfolge, wie sie einem in den Sinn kommen, z. B. bei einer To-do-Liste) oder situationsabhängig sein (Diskussionsprotokoll, das Elemente aufnimmt, wie sie im Gesprächsverlauf angesprochen wurden) (vgl. Fludernik 2016, S. 209f.).

c) Funktion »One does not read but uses lists « merkt Doležalová (2009b, S. 1) an - Listen, insbesondere praktische Listen haben eine pragmatische Funktion, einen

\footnotetext{
12 Ledins (2015) semiotisches Modell zur >Liste und Tabelle< ist für vorliegenden Zweck leider nicht weiter anwendbar, da es nicht zwischen Liste und Tabelle differenziert. Seine drei >semiotiska principer för meningsskapande< (semiotische Prinzipien zur Bedeutungsschöpfung, Übersetzung MW), >Kategorisierung «, >Interaktion` und >Informationswert ` beziehen sich vorderhand auf die Tabelle, d. h. eine komplexe Liste mit paradigmatischen, vertikalen und syntagmatischen, horizontalen Beziehungen (vgl. Mainberger 2015, S. 265 f., die definitorische Abgrenzung von >Tabelle < und >Liste < wird in Ledin 2015 nicht geleistet).
} 
Anwendungszweck, und werden dieser Anwendung gemäß von Akteuren erstellt und arrangiert. Die Form einer Liste wie auch Kohärenz und semantische Ordnung sind somit nach dem Zweck gerichtet. Der Zweck wird je nach sozialem Kontext und kulturellem, beziehungsweise institutionellem Rahmen bestimmt und kann beispielsweise auf das Auffinden und Konsultieren von Informationen ausgerichtet sein, zur Inventierung von Gegenständen, Dokumentation von Ereignissen, administrativen Verwaltung, beziehungsweise als Erinnerungshilfe oder Plan für zukünftige Ereignisse, z. B. den Einkauf oder eine Reise, intendiert sein.

\section{Namenlisten in Libri vitae}

\subsection{Formale Eigenschaften}

Die Namenlisten in Libri vitae befinden sich grundsätzlich in gebundenen Codices, wobei einige Handschriften geplant als Verbrüderungsbücher angelegt (siehe oben, Abschnitt 2) und formal so gestaltet sind, dass der Schriftraum einer Handschriftenseite oder Teile davon jeweils für die Liste einer bestimmten sozialen Kategorie, z. B. jene aller lebenden Mönche des Klosters St. Gallen (vgl. Abschnitt 4.2), vorgesehen ist. Dabei wurde jeweils in der Anlage Platz frei gelassen, um die Listen über weitere Jahrzehnte weiterzuführen. Je nach Gewichtung einzelner Kategorien standen auf einzelnen Seiten mehr oder weniger Raum für Nachträge offen - so überstieg beispielsweise sowohl im Reichenauer als auch im jüngeren St. Galler Verbrüderungsbuch der freigelassene Platz für laikale Kategorien jenen für die klerikalen um ein Mehrfaches (Geuenich 2015, S. 141; Ludwig 2015, S. 172, siehe Abschnitt 4.2).

Neben dieser planmäßig in die Codices integrierten Materialität der Namenlisten kommen zwei weitere Formen von Schriftträgern vor, die allerdings nur vereinzelt belegt sind. Zum einen gibt es Namenlisten auf losen Einzelblättern, die den Einträgen in die Codices als Vorlage dienten und von denen die Namen kopial in die Handschriften übertragen wurden. Zum andern sind kleinere Zettel mit Namenlisten überliefert, die entweder ebenso in die Handschrift kopiert oder materiell in den Codex integriert wurden. Karl Schmid und Otto Gerhard Oexle (1974 in Geuenich 2015, S. $128 \mathrm{f}$.) konnten feststellen, dass Listen auf losen Einzelblättern die ursprüngliche Form der Verbrüderung darstellen und solche Namenlisten in der Folge des Gebetsverbundes von Attigny 762 zwischen den Klöstern ausgetauscht wurden. Diese Listen mussten in Reichenau bis zur Entstehung des Codex um 824 aufbewahrt worden sein, was sich darin zeigt, dass gewisse Mönchslisten, die in der Anlageschicht des Verbrüderungsbuchs aufgenommen wurden, Namen aufweisen, die bis zu 50 Jahre zurückreichen (vgl. Geuenich 2015, S. 129). Während in Reichenau keine einzelnen Namenlisten erhalten sind, verzeichnet das St. Galler Verbrüderungsbuch den glücklichen Fall, dass eine solche in die Handschrift übertragene Liste, jene mit dem Verzeichnis der Mönche aus Nonantola, später als loses Doppelblatt in den Codex miteingebunden wurde (pag. 27-30, Abschrift auf pag. 61-64; Schmid 1986, S. 225-228, 139, 141-144; vgl. Ludwig 2015, S. 165). Auch für andere Libri vitae kann angenommen werden, dass nicht Handschriften zur Ab- 


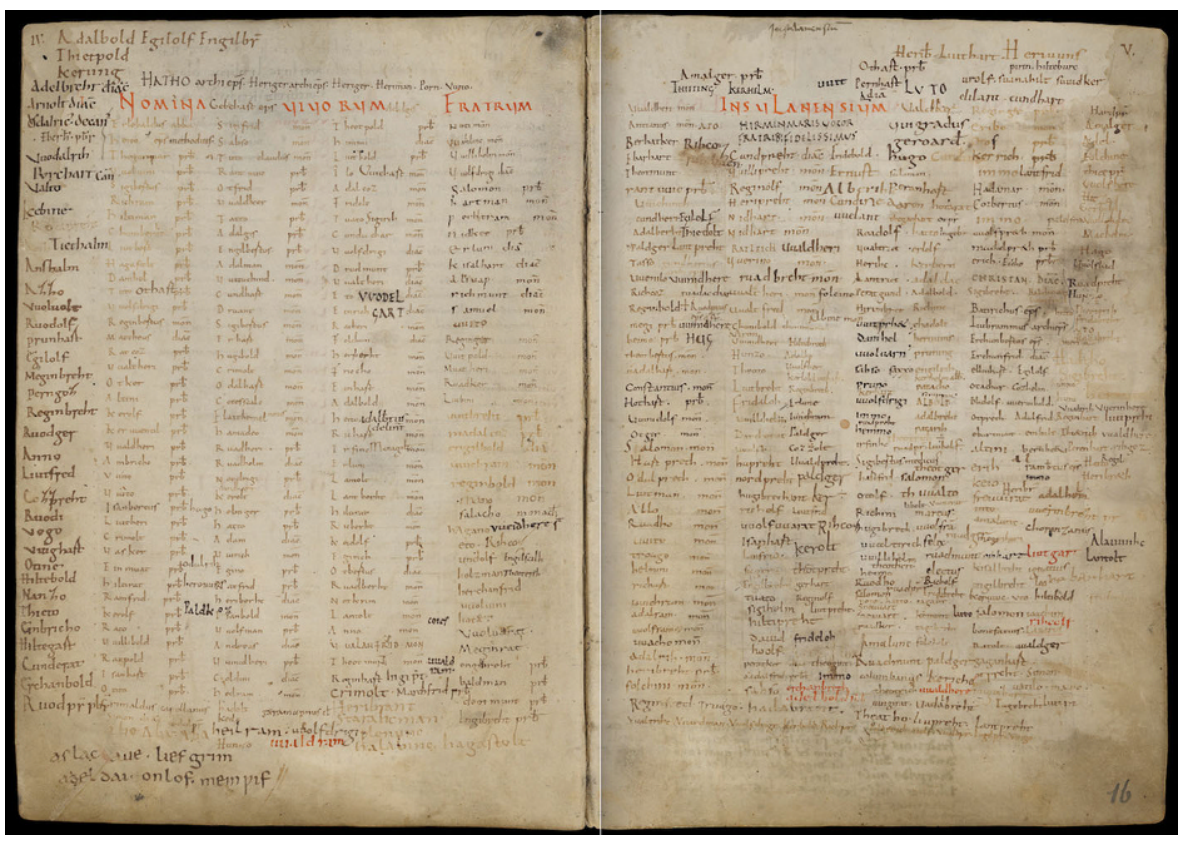

Abb. 1 Doppelseite aus dem Reichenauer Verbrüderungsbuch (Zürich, Zentralbibliothek, Ms. Rh. Hist. 27 , fol. $15 \mathrm{v}$ und fol. $16 \mathrm{r}$, www.e-codices.ch)

schrift von Namenlisten unter den Klöstern zirkulierten, sondern Listen auf losen Einzelblättern. Selbst für spätere Einträge von weiteren Namengruppen beispielsweise von vorbeireisenden Pilgern, die von lokalen Schreibern in der Handschrift notiert wurden, besteht die Möglichkeit, dass sie zum Teil von vorgeschriebenen Listen auf Zetteln kopiert wurden, auch wenn für einige dieser Einträge eine mündlich übertragene Verschriftung außer Zweifel steht (vgl. Waldispühl im Druck). Eine Vorstellung des Umfangs und der Größe solcher Zettel geben einige eingebundene Exemplare im Salzburger Verbrüderungsbuch (vgl. Diesenberger 2010, S. 33) sowie die im Reichenauer Verbrüderungsbuch auf später eingebundenen, vorangestellten Seiten eingeklebten Papierstreifen (z. B. auf fol. 11r) aus dem 14.-16. Jahrhundert und die in derselben Handschrift in einer Papiertasche auf der hinteren Innenseite gesammelten Exemplare.

In der formalen Gestaltung der Einzelseiten zeigen die Libri vitae einige Gemeinsamkeiten, jedoch überwiegen die Unterschiede und keine Handschrift gleicht exakt der anderen. Während im Reichenauer Codex die Namen einer bestimmten sozialen Kategorie in der Anlageschicht aus dem ersten Drittel des 9. Jahrhunderts zumeist auf einer einzelnen oder einer Doppelseite (z. B. fol. 15v und fol. 16r, s. Abb. 1) verteilt auf vier blindliniierte Kolumnen notiert und die Kategorie hyperonymisch in roter Tinte überschrieben wurde, liegt zwar auch in anderen Handschriften die vertikale Kolumne als grundsätzliches formales Gestaltungsprinzip des Schriftraums vor, jedoch wurden diese nicht selten elaborierter mit Arkaden ausgestaltet, in deren Zwischenräume die Namenlisten zu stehen kamen. Ein Beispiel sind die beiden 
Abb. 2 Älteres St. Galler Verbrüderungsbuch (Stiftsarchiv St. Gallen, StiASG, C3 B 55, pag. 8)

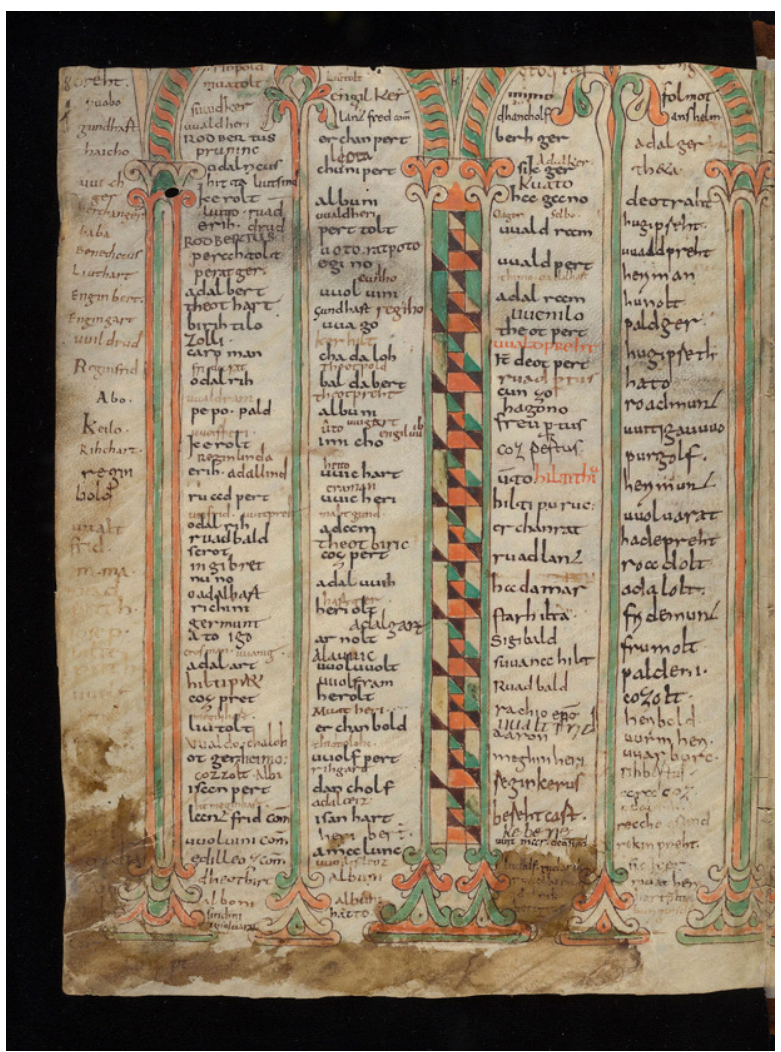

St. Galler Verbrüderungsbücher, wobei im älteren die Doppelarkaden koloriert und kunstvoll gestaltet sind (z.B. pag. 8 und 9, s. Abb. 2; Abb. 3), im jüngeren allerdings in schlichterer Form und einfacher roter Federstrichzeichnung (z. B. pag. 49, s. Abb. 4) erscheinen und im hinteren Teil zwar als Struktur blindliniiert, jedoch nicht mehr ausgestaltet sind (pag. 77-78). Arkaden und Säulen als Gestaltungselemente der Manuskriptseiten finden sich ebenso in den Libri vitae von Salzburg, Pfäfers und Remiremont. Die insularen Codices hingegen sind einfacher gestaltet, halten sich jedoch an die Kolumne als Gestaltungsprinzip.

In den Anlageschichten der jeweiligen Handschriften wird diese vertikale Anordnung der einzelnen Namen strikt eingehalten. Es zeigen sich jedoch auch in Bezug auf dieses grundlegende formale System innerhalb einer Handschrift Unterschiede. So sind die einzelnen Nameneinträge auf fol. $15 \mathrm{v}$ im Reichenauer Verbrüderungsbuch beispielsweise innerhalb einer Kolumne formal in jeweils drei weitere Kolumnen unterteilt: Die Nameninitialen in Majuskeln stehen in einer Reihe vertikal untereinander, in einer weiteren Kolumne folgt der Rest des Namenkörpers und in einer dritten steht das abgekürzte, klerikale Attribut (z.B. presbiter, monachus, diaconus). Auf anderen Seiten werden diese drei Namenelemente horizontal enger aneinandergesetzt und der Rufnamenkörper nicht getrennt. 
Abb. 3 Älteres St. Galler Verbrüderungsbuch (Stiftsarchiv St. Gallen, StiASG, C3 B 55, pag. 9)

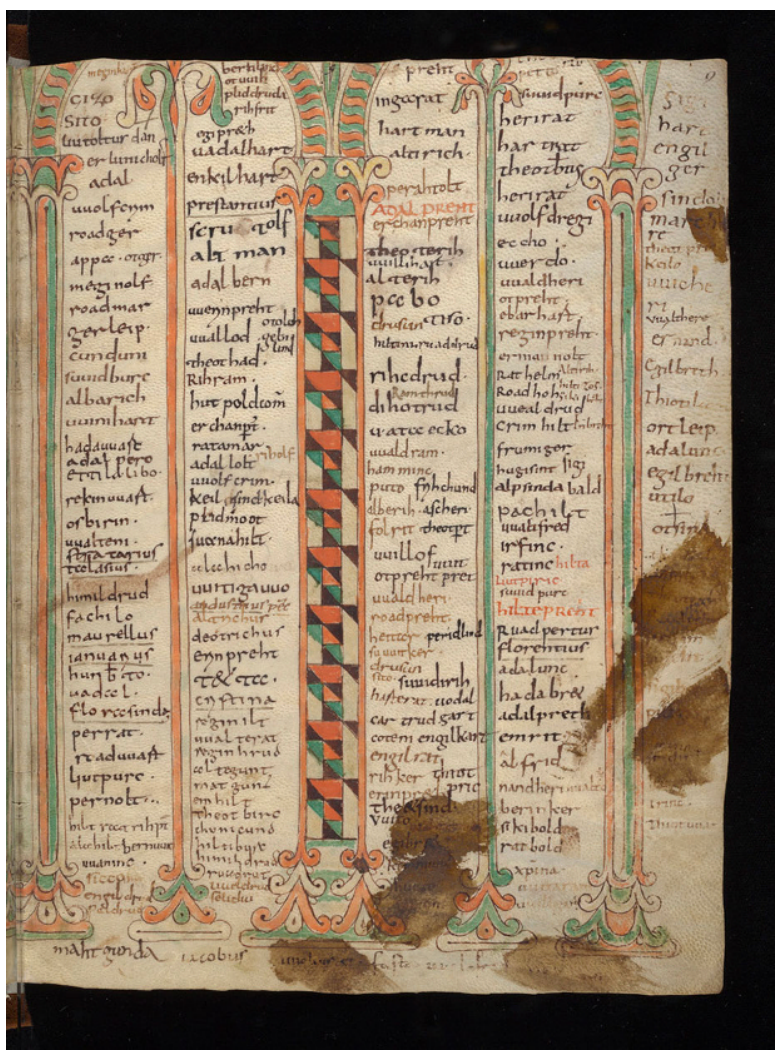

Für mehrere Handschriften fällt auf, dass das ursprüngliche Layout der Anlageschicht bei späteren Einträgen nicht mehr beachtet und auch die Marginalien und frei gebliebenen Räume zwischen ursprünglichen Kolumnen beschriftet wurden (vgl. z. B. Reichenau, fol. 15v und 16r, s. Abb. 1).

Spätere Einträge kamen im Reichenauer Verbrüderungsbuch auch auf Einzelblättern zu stehen, deren Spaltenaufteilung weniger deutlich erkennbar ist (vgl. z. B. fol. 93, Autenrieth 1979, S. XXI). In diesen Einträgen wird oft dennoch das vertikale Prinzip eingehalten und die Namen stehen aneinandergereiht in einem Block mit jeweils einem Namen pro Zeile. Daneben gibt es allerdings auch Listen mit mehreren Namen pro Zeile (fol. 93r Liste oben rechts), die auch mittels Punkten voneinander getrennt werden (fol. 93r, »hislant terra«-Liste oben links) und selbst die horizontale, am Lauftext orientierte Anordnung mit Wortabstand oder mit Punkt kommt vor (vgl. z. B. fol. 89r oben, fol. 93v, fol. 94r, fol. 94v).

$\mathrm{Zu}$ den formalen Eigenschaften gehören schließlich die Eintragungstechnik sowie der Schrifttyp und die Schriftgestaltung. Meines Wissens stellen die Namenlisten generell Tinteneinträge dar. Dass überhaupt auch mit anderen Schreibwerkzeugen Eintragungen angebracht wurden, konnte Peter Erhard (2010, S. 49f.) jüngst am St. Galler Verbrüderungsbuch zeigen. Im Zuge kürzlich vorgenommener Restaurierungsarbeiten kamen bisher unbekannte Griffeleinträge zum Vorschein. Es handelt 
Abb. 4 Jüngeres St. Galler Verbrüderungsbuch (Stiftsarchiv St. Gallen, StiASG, C3 B 55, pag. 49)

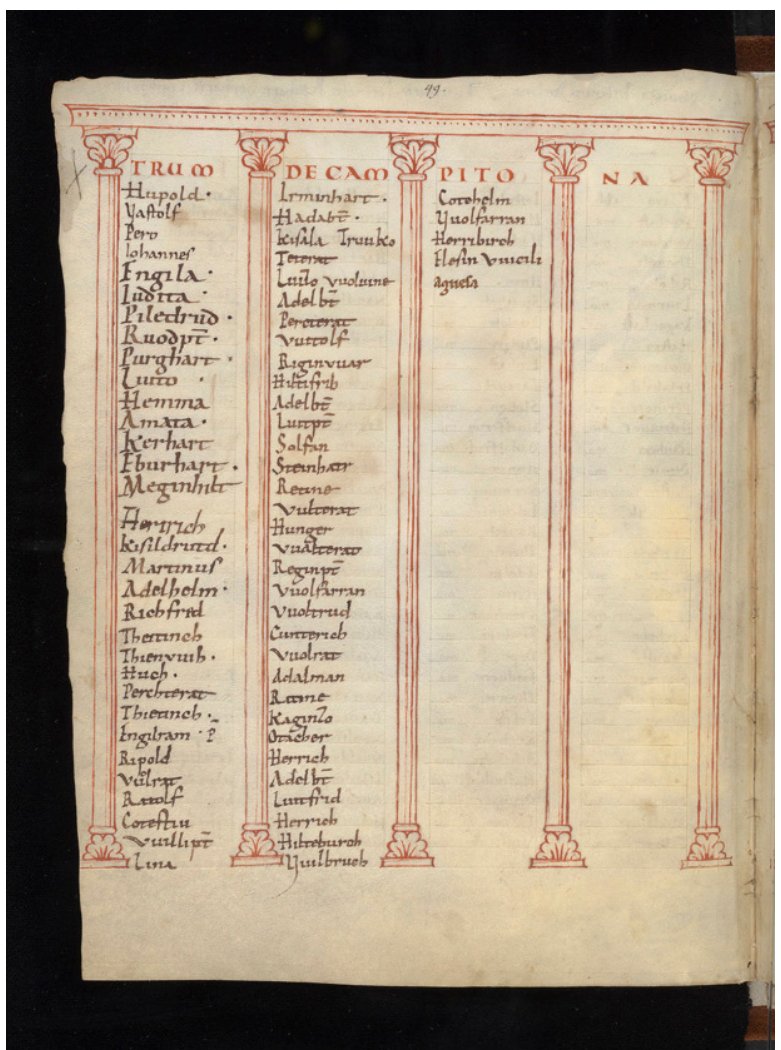

sich zumeist um Einzeleinträge außerhalb von Listen, auf p. 18 wurde jedoch auch eine Liste von mehreren mit Griffel eingeritzten, allerdings schwer zu entziffernden Namen entdeckt, die über vorgängig radierte Tinteneinträge eingetragen wurde. ${ }^{13}$

Der Schrifttyp und dessen Ausführung stellt schließlich in der bisherigen geschichtswissenschaftlichen Erschließung der Libri vitae eine der wichtigsten methodischen Errungenschaften dar. Der Schrifttyp und dessen paläographische Beschreibung bilden die Ausgangslage dafür, zusammengehörige Listen, d.h. von einer Schreiberhand in einem Zug eingetragene Namengruppen (vgl. Schmid 1979, S. LXXXI) zu ermitteln und chronologisch zu bestimmen. Außerdem können Modifikationen an ursprünglichen Listen und spätere Ergänzungen paläographisch festgestellt werden, was für die kulturelle Deutung von zentraler Bedeutung sein kann (vgl. zum Liber viventium Fabariensis im Abschnitt 4.2). Die Veränderung der oben erwähnten Layoutpraktiken korreliert in allen Verbrüderungsbüchern mit der steigenden Anzahl unterschiedlicher Schreiberhände, die ergänzende Eintragungen in späterer Zeit vornahmen. Manche Schreiber stammten wohl gar von außerhalb, wie

13 Da ein Großteil der Editionen der Libri vitae aus einer Zeit stammen, in der die Griffelglossenforschung noch weniger etabliert war als heute, ist nicht auszuschließen, dass bei erneuter Untersuchung der jeweiligen Handschriften weitere Glosseneinträge entdeckt werden. 
dies Diesenberger (2010, S. 34) für das Salzburger Verbrüderungsbuch annimmt, bei dem einige Hände im Salzburger Skriptorium sonst nirgends bezeugt sind.

Von der nicht-intendierten Ausführung des Schrifttyps einzelner Schreiber gilt es, die intendierte Schriftgestaltung beispielsweise mittels Kolorierung oder Majuskelschreibung abzugrenzen. Diese drückt meist eine Hervorhebung des jeweiligen Eintrags aus und kommt vorwiegend in Überschriften zur Anwendung. In der Anlageschicht des Durham Liber vitae wurde die Kolorierung allerdings als ästhetisches Gestaltungsprinzip angewandt, indem die Namen abwechslungsweise in schwarzer, silberner und blauer Tinte eingetragen wurden. In einem weiteren Sinn hierher gehört zudem die Wahl des Schriftsystems. Im Reichenauer Verbrüderungsbuch gibt es einen Eintrag in griechischen Majuskeln (fol. 40v) und einen weiteren in insularen Minuskeln (fol. 48v) (vgl. Geuenich 1979, S. LIX). Beide Einträge finden sich unter späteren Ergänzungen und zeugen von autographen Eintragungen vorbeireisender Pilger.

\subsection{Kohärenzen und innere semantische Ordnungen}

In der Listenform der Libri vitae sind die Namen nach bestimmten inhaltlichen Kriterien kategorisiert, wodurch sowohl verschiedene Ordnungen des Sozialen in der frühmittelalterlichen Gesellschaft als auch kulturhistorische und politische Zusammenhänge dargestellt, beziehungsweise hergestellt werden. Im Folgenden soll aufgezeigt werden, welche semiotischen Eigenschaften der Liste in den Libri vitae genutzt werden, um soziale und kulturelle Kategorien zu vermitteln und wie unterschiedliche Kohärenzen erschlossen und interpretiert werden. Dieser Abschnitt thematisiert somit erst einmal die inhaltlichen Zusammenhänge der einzelnen Listenelemente, während im nächsten Abschnitt 4.3 auf die pragmatischen Funktionen der Kategorisierungen und Darstellungsweisen eingegangen wird.

Die Namenlisten der Anlageschichten aus dem 9. Jahrhundert sind, wie schon erwähnt, strikt nach sozialen Kategorien geordnet aufgeführt. Im Reichenauer Verbrüderungsbuch kamen diese Kategorien formal jeweils auf einzelnen Seiten zu stehen, die zudem mit Peritexten wie Farbüberschriften, welche die Kategorisierung sprachlich wiedergaben und typographisch offenbar machten, versehen wurden. Zusätzlich wurde diese Kategorisierung in einem Inhaltsverzeichnis (capitula) auf fol. $15 r$, einer Liste mit Metafunktion, wiedergegeben. Ein solches Verzeichnis ist für Libri vitae einzigartig. Die Überschriften sind hingegen auch in anderen Codices vorhanden (z. B. älteres und jüngeres St. Galler Verbrüderungsbuch). Der Hauptanteil der Kategorien machen Konvente aus, z. B. nomina fratrum de monasterio quod vocatur leonis. Im Reichenauer Verbrüderungsbuch wurden die Angehörigen einiger Konvente weiter nach lebenden (nomina vivorum fratrum insolanensium) und toten (nomina defunctorum fratrum insolanensium) eingeteilt, dasselbe gilt für das Salzburger Verbrüderungsbuch. Von den klerikalen Kategorien getrennt aufgeführt sind die laikalen Kategorien der Freunde und Gönner, die in Reichenau wiederum in lebende und verstorbene unterteilt wurden, in St. Gallen hingegen in weibliche und männliche.

Die Reichenauer Liste der Anlageschicht mit der Überschrift nomina amicorum viventium (fol. 62v) zeigt zusätzlich eine innere Strukturierung nach den ordines der 
frühmittelalterlichen ständischen Gesellschaftsordnung in Königsfamilie, Bischöfe, Äbte, Priester und Grafen, dann weitere Laien verteilt auf die vier liniierten Kolumnen (vgl. Zettler 2010, S. 67, Geuenich 2015, S. 135). In der Liste der verstorbenen Wohltäter wurden allerdings die beiden mittleren Spalten leer gelassen, während die erste Spalte Mitglieder der karolingischen Königsfamilie und die letzte einige Grafen nennt. Die Angehörigen der alemannischen Herzogsfamilie wurden in der zweiten Spalte der nächsten Seite platziert, worauf weitere Grafen folgen (vgl. Zettler ebd.). Diese Kategorisierung ist jedoch nicht hyperonymisch überschrieben, sondern kann nur historisch erschlossen werden, indem die Namenträger mit Daten aus anderen historischen Quellen in Verbindung gebracht und sozial kontextualisiert werden können. Dieselbe Erschließungsmethode gilt auch für die Einträge der Anlageschicht im Liber viventium Fabariensis auf p. 24 und 25, wo einerseits wie in Reichenau Angehörige des weltlichen Adels (auf p. 24) andererseits Bischöfe und Priester (auf p. 25) verzeichnet sind. Hier wird allerdings bei diversen Namen ein Zusatz rex, imperator, laicus, episcopus oder presbiter beigestellt, womit die soziale Zugehörigkeit lexikalisch angegeben wird. Auch im Salzburger Verbrüderungsbuch wurde in der ältesten Schicht die Einteilung nach ordines vorgenommen, allerdings sind den klerikalen und weltlichen Kategorien zusätzlich noch Namen aus dem alten und neuen Testament vorangestellt (pag. 5). Als zusätzliches Kohärenzprinzip kommt hinzu, dass Mönche einem Lehrer zugeordnet wurden (Diesenberger 2010, S. 31, 34).

Eine weitere inhaltliche Kategorie, die nicht explizit sprachlich gekennzeichnet wurde, jedoch aber nicht historisch, sondern onomastisch erschlossen werden kann, sind getrennte Listen für Männer und Frauen, wie z. B. die Aufteilung der männlichen Angehörigen von Karolingern und weiteren weltlichen Adligen in der linken und der weiblichen in der rechten Spalte auf p. 24 im Liber viventium Fabariensis (vgl. Lieven 2010, S. 84). ${ }^{14}$ Diese schrifträumliche Trennung vermittelt somit gesellschaftliche Zugehörigkeit der weltlichen Adligen nach Geschlecht, das neben den ständischen ordines offenbar eine zentrale soziale Kategorie darstellte. ${ }^{15}$

Im jüngeren St. Galler Verbrüderungsbuch, das ursprünglich als Abschrift des älteren St. Galler Verbrüderungsbuches entstanden ist (Schmid 1986, S. 213-241, zusammenfassend vgl. Ludwig 2015, S. 147f. ${ }^{16}$ wurden die Kategorien »klerikal« vs. »laikal«, die schon im älteren Verbrüderungsbuch räumlich auf unterschiedlichen Seiten getrennt aufgeführt und mit Überschriften gekennzeichnet sind, in der Abschrift dadurch verstärkt, dass jedem Namen systematisch der Zusatz monachus und laicus beigestellt wurde. Die Trennung dieser beiden Kategorien scheint für die beiden St. Galler Codices von entscheidender funktionaler Bedeutung, im älteren Verbrüderungsbuch wurde ihr selbst in den späteren Nachträgen strikt Folge geleistet: Obwohl auf den Seiten der Laieneinträge der freie Platz knapp wurde

\footnotetext{
14 In Lieven (2010, S. 84) ist >links < und >rechts< versehentlich vertauscht. Ein Blick auf die Abbildung 39 stellt den Irrtum jedoch klar.

$15 \mathrm{Zu}$ Geschlechterpaaren als inneres Ordnungsprinzip vgl. weiter unten.

16 Für das ältere Verbrüderungsbuch wird eine Entstehungszeit um 807-817 angenommen (vgl. Ludwig 2015, S. 158), für das jüngere um 860 (Ludwig 2010). Die beiden Bücher sind später in einem Codex zusammengebunden worden (vgl. Schmid 1986, S. 213-241).
} 
und Namen interlinear und marginal in immer kleineren Schriftgrößen angebracht wurden (vgl. pag. 8-17), wich man nicht auf die Seiten der Klerikaleinträge aus (vgl. Ludwig 2015, S. 163 f.). Anders im Reichenauer Verbrüderungsbuch, wo zwar anfangs die Namen zu einzelnen Kategorien systematisch nachgeführt wurden Zettler (2010, S. 68) spricht von einer »richtiggehenden Buchführung « - die ursprüngliche Kategorisierung im Verlauf der späteren Fortführung der Listen jedoch schlichtweg überschrieben wurde. Ebenso wird im Liber viventium Fabariensis die Trennung von Frauen- und Männernamen bei den Nachträgen nach der Mitte des 9. Jahrhunderts nicht mehr eingehalten (Lieven 2010, S. 87).

Die Namen in einer Liste können auch etymologisch eine Einheit bilden. Nach diesem Kriterium wurde die Gruppe von über 700 Namenbelegen »nordischer Spezifik « (Naumann 1992, S. 705) im Reichenauer Verbrüderungsbuch bestimmt, die auf mehrere Seiten verteilt in über 20 verschiedenen Listen stehen. Abgesehen von einer Liste mit der Überschrift hislant terra sind die Listen allerdings in der Handschrift nicht explizit kategorisiert, sondern die Zusammengehörigkeit wurde aus dem onomastischen Sprachmaterial erschlossen. Diese Nameneinträge gehören zu den späteren Ergänzungen, die im 11.-12. Jahrhundert in der Handschrift notiert wurden (zur Datierung vgl. Jørgensen/Finnur Jónsson 1923). Kulturhistorisch wird dieses Namenkorpus im Zusammenhang mit Pilgerreisen nordischer Gläubiger nach Rom oder gar Jerusalem gedeutet, die auf ihrem Weg das Reichenauer Kloster besuchten und da ihre Namen hinterließen (vgl. Jørgensen/Finnur Jónsson 1923). Innerhalb dieses Teilkorpus zeigt sich im Übrigen ein interessanter Fall einer Überlappung der bisher in Bezug auf formale und inhaltliche Listenzusammengehörigkeit definierten Kriterien. Im Listeneintrag in den Spalten $C^{2-5}$ und $X^{1-4}$ auf fol. 89r (p. 151) widersprechen sich die paläographische und die etymologische Zusammengehörigkeit der Namen. Die Mehrheit der insgesamt 62 Namenbelege, die von derselben Hand eingetragen wurden (Naumann 1992, S. 725), ${ }^{17}$ kann etymologisch als nordgermanisch bestimmt werden und ist zudem in zeitgenössischen, nordischen Quellen verbreitet, z. B. Toki, Asa, Eskel. In derselben Liste finden sich hingegen auch deutsche Namen, etwa Ruodolf und Pertolt. Ob dieses Faktum auf eine »gemischte « Pilgergruppe, die zusammen gereist ist, hindeutet oder ob der Eintrag vielmehr auf einen Schreibvorgang zurückgeht, bei dem ein Schreiber mehrere einzelne Listenzettel in einem Prozess in den Codex kopiert hat, kann mangels Überlieferung allfälliger Zettel nicht beantwortet werden. Bemerkenswert an der Liste sind allerdings zwei nordgermanische Anthroponyme, Halbtene und $\mathrm{Ka}(r)$ leshoub(it), deren Namenglieder ins Mittelhochdeutsche übertragen wurden - was auf ein Bewusstsein für Mehrsprachigkeit hindeuten und das Argument einer gemischten Reisegruppe unterstützen könnte (vgl. Waldispühl 2018, S. 148). Dieses Fallbeispiel zeigt die Vorteile beziehungsweise Notwendigkeit einer interdisziplinären Analyse zur kulturhistorischen Erschließung der Nameneinträge deutlich auf.

Ein Beispiel zur politischen Deutung etymologischer Zusammengehörigkeit gibt Walter Kettemann (2010, S. 90) anhand der Namen der Bischofsliste im Liber viventium Fabariensis sowie der Konventsliste des Klosters Müstair im älteren St. Galler

17 Bei Naumann (1992, S. 725) fehlen allerdings für ebendiesen Eintrag die drei Namenbelege Zure, Suuein, Truith zwischen Truith und Ka(r)leshoub(it) in der Spalte X, vgl. Waldispühl (2017, S. 265). 
Verbrüderungsbuch aus der Mitte des 9. Jahrhunderts. In der Bischofsliste sind bis zum Jahr 849 lateinische oder lateinisch-romanische Namen verzeichnet, danach dominieren germanische Namen, während in der Müstair-Liste von insgesamt 44 Namen 40 eine lateinische, lateinisch-romanische, griechische oder hebräische Etymologie aufweisen, der als erstes genannte Abt jedoch einen germanischen Namen trägt. Diese Aufteilung von lateinisch, lateinisch-romanischen auf der einen Seite und germanischen Namen auf der anderen Seite deutet laut Kettemann (2010, S. 90) auf personalpolitische Maßnahmen im Zuge der Machtausbreitung Ludwig des Deutschen in Rätien hin.

Nachdem die bisherigen Betrachtungen zur Kohärenz in den Libri vitae den übergreifenden, paradigmatischen Listenkategorien galten, die einzelne Namen zu einem Ganzen verbinden, soll im Weiteren die innere Gliederung einzelner Listen zur Sprache kommen. Denn unter den Namenlisten in Libri vitae lassen sich mehrere finden, in denen die einzelnen Namen nach einer bestimmten Abfolge aneinandergereiht sind. Ein erstes Ordnungsprinzip, das mehrfach vorkommt ist die chronologische Reihung. Die dem Reichenauer Konvent angehörigen Mönche in der Totenliste beispielsweise sind laut Zettler (2010, S. 62) seit ca. 780 in der Reihenfolge verzeichnet, wie sie gestorben sind. Er stellt gar fest, dass einige der in der Totenliste nachgetragenen Namen auch in der Liste der lebenden Reichenauer Mönche vorkommen, die beiden Listen sich demnach überschneiden (Zettler 2010, S. 63). Anders im Salzburger Verbrüderungsbuch, wo die Namen verstorbener Mönche aus der Liste der Lebenden radiert und überschrieben wurden (Diesenberger 2010, S. 33). Auch die Nameneinträge der Churer Bischöfe auf p. 25 im Liber viventium Fabariensis folgen einer chronologischen Abfolge - allerdings wurde diese bewusst manipuliert, wie Walter Kettemann (2010, S. 92) aus dem paläographischen Befund schlussfolgert. Die Namen von zwei Bischöfen, Victor III. und Verendarius, wurden zuerst im Anschluss an vier schon bestehende Namen (Victor pre, Tello eps, Canstrantius pre und Remedius eps) eingetragen, zu einem späteren Zeitpunkt jedoch getilgt und überschrieben mit den Namen Verendarius eps und Esso eps. Der Name Victor III. wurde folglich aus der Liste entfernt. Kettemann (2010, S. 92) deutet diese »damnatio memoriae« politisch, indem er Victor III. als Repräsentanten einer älteren bischöflichen Politik versteht, die mit den Veränderungen in Rätien unter Ludwig dem Deutschen um 850 nicht mehr vereinbar war. In der Anordnung der Namen und deren Modifikation würde sich somit ein zeitgenössischer Entwurf eines Geschichtsbildes von Churrätien mit einer »dezidierten geschichtspolitischen Konzeption« offenbaren (Kettemann 2010, S. 93). Es versteht sich von selbst, dass - ebenso wie bei den nicht explizit sprachlich ausgedrückten Kategorien oben - historisches und kulturelles Wissen vorausgesetzt ist, um diese inneren Abfolgen der Listen dieser Art zu deuten.

Eine weitere Art der inneren Gliederung zeigt sich in einer der Listen $\left(\mathrm{A}^{2}-\mathrm{D}^{3}\right.$ fol. 94v) der nordgermanischen Namen im Reichenauer Verbrüderungsbuch. Diverse Namenpaare mit jeweils einem Männer- und einem Frauennamen kommen vor, in einem Fall, thore octhora, sind diese beiden Namen gar mittels der Konjunktion altnordisch $o c$ >und < miteinander verbunden. Diese Anordnung lässt auf Ehepaare schließen, die sich zumindest zusammen eintragen lassen haben, eventuell gar miteinander gereist sind (vgl. Naumann 1992, S. 721). 
Diverse innere Abfolgen in Namenlisten sind allerdings bisher ungedeutet, insbesondere die Laieneinträge, die nach der Anlageschicht auf den ursprünglich leer gelassenen Seiten von vielen verschiedenen Schreiberhänden ergänzt wurden (vgl. Zettler 2010, S. 67, Geuenich 2015, S. 140f. für das Reichenauer Verbrüderungsbuch).

\subsection{Pragmatische Funktionen}

Die Libri vitae waren über Jahrzehnte, beziehungsweise gar über Jahrhunderte >lebendige< Textdokumente - die Bücher waren in Gebrauch und Namen wurden laufend ergänzt. Diese historische Dimension brachte formale und inhaltliche Änderungen mit sich. Die mittels konzipierter Anordnung, Peritexten und deutlich voneinander abgegrenzter, sozialer Kategorien strukturierten älteren Listensammlungen gingen in >Rhizome< ohne Anfangs- und Endpunkt (vgl. Eco 2009, S. 238) mit marginalen und interlinearen Namenergänzungen über, in denen der älteren Ordnung nicht mehr strikt oder überhaupt nicht mehr Folge geleistet wurde. In der geschichtswissenschaftlichen Forschung wurde dieser formale und inhaltliche Wandel pragmatisch gedeutet, d.h. es wird angenommen, dass sich die Funktion und der Gebrauch der Libri vitae bei deren Anlage im späten 8. und Anfang/Mitte 9. Jahrhundert im Vergleich zu ihrer späteren Verwendung verändert hat.

Der frühe Gebrauch steht im Zeichen der monastischen Gebetsverbrüderung, die im Gebetsbund von Attigny zwischen verschiedenen kontinentalen Klöstern vereinbart wurde (vgl. Geuenich 2015, S. 130 mit Literatur, vgl. auch Lieven 2015, S. 247-251). Die formale und inhaltliche Struktur der Codices wurde diesem Gebrauch gemäß angelegt: Die Listen sollten die vereinbarte Gebetsverbrüderung zum Ausdruck bringen - deshalb die genaue Kategorisierung nach Konventszugehörigkeit. Außerdem bestand die Absicht, jeweils weitere Mitglieder hinzuzufügen und in diese Struktur zu integrieren. Für diese Funktion boten die Überschriften und beim Reichenauer Exemplar das Inhaltsverzeichnis Orientierung. Mit diesen strukturellen Hilfsmitteln konnte die korrekte Stelle im Codex einfacher nachgeschlagen werden (vgl. Geuenich 2015, S. 130). ${ }^{18}$ Die Listenfunktion ist allerdings in dieser älteren Zeit schon vielfältig. Bei der Anlage stand die Verbrüderung der Klöster im Vordergrund und die Listen dienten als materielle Verfestigung des gegenseitigen Gebetsgedenkens in Anlehnung an die in der Offenbarung des Johannes erwähnten Libri vitae. Die Namen der Klosterangehörigen wurden allerdings zuerst auf losen Zetteln (vgl. Abschnitt 4.1) gesammelt, beziehungsweise inventiert, bevor sie in die Codices übertragen wurden. Dadurch, dass bei der Abschrift in den Codices Platz für Nachtragungen freigelassen wurde, bekamen die Listen eine zusätzliche administrative Dimension. Es wurde »buchgeführt« über neu eintretende Mönche, und verstorbene Angehörige wurden von der Lebenden- in die Totenliste überführt. Die Listen wurden editiert und redigiert um der Kategorisierung Folge zu leisten.

$18 \mathrm{Im}$ Inhaltsverzeichnis sind allerdings nicht alle Listen erfasst und auch unter den aufgenommenen sind die letzten drei nachgetragen, was bedeutet, dass einige Listen nach der Anlage des Codex eingetroffen sind (Geuenich 2015, S. 130). 
Die primäre Funktion der Gebetsverbrüderung ging später über in jene eines liturgischen Gebetsgedenkens, bei dem das Laiengedenken gegenüber dem monastischen immer mehr an Bedeutung gewann (vgl. Schmid 1989, S. 134 f.). Dies zeigt sich darin, dass die ursprüngliche Kategorisierung nicht mehr eingehalten wurde, d.h. z. B. Männernamen in der Frauenliste zu stehen kamen (vgl. oben), und die Namen später schlicht da eingetragen wurden, wo sich Platz fand. Während diese Deutung insbesondere für das Reichenauer Verbrüderungsbuch wie auch für den $\mathrm{Li}$ ber viventium Fabariensis und den Salzburger Codex geltend gemacht werden kann, betont hingegen Ludwig (2015, S. 163, 172f.), dass der großzügig angelegte Platz für die laikalen Einträge in den St. Galler Verbrüderungsbüchern und die strikte Trennung dieser Einträge von den klerikalen auch bei der Fortführung der Listen darauf hinweise, dass das Laiengedenken in der St. Galler Memoria von Anfang an einen zentralen Platz einnahm.

Mit dem funktionalen Wandel der Libri vitae im Verlauf des 9. Jahrhunderts verändern sich auch die Schreibpraktiken - mehrere Hände nehmen Eintragungen vor, Einzelnamen außerhalb von Listen und autographe Eintragungen treten vermehrt in Erscheinung. Die in der Anlageschicht festgelegte inhaltliche Kohärenz als Ordnungsprinzip der Liste verliert ihre Bedeutung, beziehungsweise sie verschiebt sich auf andere Kategorien, die zumeist nicht explizit sprachlich ausgedrückt werden. Damit erschwert sich ihre Erschließung. Fehlt eine onomastische Einheitlichkeit, kann eine allfällige Kohärenz räumlich zusammenstehender und von einer Hand eingetragener Namen nur über außersprachliches Wissen erfasst werden. Für die pragmatische Funktion des liturgischen Gedenkens sollte jedoch nicht ausgeschlossen werden, dass die Kohärenz sowohl aus Sicht des/der mit Namen Vermerkten als auch des Konvents schlicht keine Rolle spielte. Die Materialisierung des eigenen Namens im Liber vitae könnte den zentralen Akt dargestellt haben, der Kontext innerhalb des Buches und die Zuordnung zu einem Kollektiv hingegen wäre als ein zu vernachlässigender Faktor gesehen worden. Die Kohärenzen, die sich in diesen späteren, paläographisch bestimmten Listeneintragungen feststellen lassen, wären somit eher auf die situativen Bedingungen des Verschriftungsprozesses zurückzuführen als auf kategoriale Prinzipien.

Im Beitrag von Kettemann (2010, vgl. oben) zum Liber Viventium Fabariensis schließlich hat sich neben dem Gebetsgedenken auch eine politische Funktion des Textdokuments gezeigt. Die Liste wurde gemäß veränderter politischer Umstände bewusst manipuliert und dient somit auch zur Manifestation und Festschreibung eines idealen Geschichtsbildes. Dieser Fall zeigt, dass die Listen in der Libri vitae schon zeitgenössisch als Quellen einer lokalen Erinnerungskultur betrachtet werden konnten.

\section{Lebendige Listen und semiotische Konstanten}

Die Namenlisten in Libri vitae kamen in unterschiedlichen Formaten auf den Handschriftenseiten zu stehen, wurden gar als separate Zettel in Codices eingebunden, bringen verschiedene Kohärenzen zum Ausdruck und erfüllen mehrere pragmatische Funktionen. Die relativ große Variation lässt sich zum einen damit erklären, dass die- 
se Dokumente über mehrere Jahrzehnte oder gar Jahrhunderte in den monastischen Betrieben als >lebendige Bücher < in Gebrauch waren - Darstellungskonventionen sowie der funktionale Rahmen und damit die Voraussetzungen für die Darstellung der Listen haben sich gewandelt. Zum anderen stellt jedes der Libri vitae ein einzigartiges Dokument dar, das im institutionellen Rahmen des jeweiligen Konvents mit der eigens definierten Gewichtung verschiedener formaler, inhaltlicher und funktionaler Aspekte angelegt und weitergeführt wurde. Die Textform Liste, die allen Handschriften zu Grunde liegt, ist in ihrer medialen Anlage so flexibel, dass sie die unterschiedlichen Funktionen wie monastische Gebetsverbrüderung, Buchführung neueintretender und verstorbener Geistlicher, liturgisches Gebetsgedenken, Manifestation eines politisch geprägten Geschichtsbildes und lokale Erinnerungskultur zu kodieren und zu vermitteln vermochte.

Trotz der Variabilität gibt es semiotische Konstanten in der Schriftraumgestaltung über mehrere Listen hinweg, die nicht nur als etablierte kulturelle Praktiken mittelalterlichen Schreibens gedeutet werden können, sondern gezielt genutzt wurden, um soziale oder politische Ordnungen zum Ausdruck zu bringen oder zu manifestieren. Eine solche Konstante, die sich gar als Grundprinzip der visuellen Anordnung festlegen lässt, zeigt sich in der Auflistung in der Vertikalen mit Beginn links oben auf einer Seite und Ende rechts unten, wobei die einzelnen Namen jeweils auf einer Zeile untereinander zu stehen kamen. Dieses Prinzip findet sich zwar in Listen aus allen Exemplaren wie auch allen Zeiten, jedoch wurde es insbesondere in den konzipierten Anlageschichten strikter eingehalten. Außerdem wurde die vertikale Anordnung in Kolumnen vorgenommen. Die Konzeption dieser räumlichen Grundstruktur zeigt sich in mehreren Codices in der Grundliniierung. Dem vertikalen und rechtsläufigen Prinzip wurde in den Anlageschichten auch da Folge geleistet, wo Platz für Ergänzungen offen gelassen wurde - dieser war grundsätzlich unten und rechts angelegt. Die Ergänzungen folgten schließlich diesem Prinzip, d.h. weitere Namen wurden nicht irgendwo in den freien leeren Raum gesetzt, sondern direkt im Anschluss an die schon bestehenden Namen eingetragen. An diesem visuellen Grundprinzip orientieren sich auch die listenimmanenten semantischen Ordnungen, die in der bisherigen Forschung festgestellt werden konnten: Hierarchische oder chronologische Abfolgen von Namen werden grundsätzlich von oben links nach unten rechts aufgeführt. Paradigmatische Kohärenzen folgten dem Grundprinzip insofern, als einzelnen Kategorien entweder ganze (Doppel-)seiten oder einzelne Kolumnen zugeordnet wurden.

Während sich die Rechtsläufigkeit als Konstante auch in den jüngeren Listen außerhalb der angelegten Kategorien zeigt, folgen diese Einträge nicht mehr strikt der vertikalen Anordnung und orientierten sich auch nicht mehr grundsätzlich am vorstrukturierten Schriftraum. Horizontal aneinandergereihte Namen kommen vor, interlineare Einträge sowie solche in den Marginalien, und bei vertikal angeordneten Listen steht gelegentlich mehr als ein Name pro Zeile. Dieser Wandel in den formalen Ordnungsprinzipien kann mit dem funktionalen Wandel der Handschriften in Verbindung gebracht werden. Die Großzahl der Libri vitae gehen von Büchern zum Zweck der monastischen Verbrüderung zu Gedenkbüchern für Laien über (vgl. Abschnitt 4.3), was dazu führte, dass die ursprüngliche Kategorisierung und entsprechende Zuordnung des Schriftraums an Bedeutung verlor. Dies führte dazu, dass 
diverse Listen beziehungsweise einzelne Namen aus unterschiedlichen Zeiten und Kontexten zufällig auf derselben Seite zu stehen gekommen sind. Ausschlaggebend war allein der noch zur Verfügung stehende offen gebliebene Platz auf einer Manuskriptseite. Während die Strukturierung des Schriftraums also in den frühen Listen semiotisch genutzt wurde, um soziale Zusammengehörigkeit zu verschriftbildlichen, verblasst diese Ausdrucksmöglichkeit in den späteren Listen und die reine Materialisierung der Namen in der Handschrift rückt in den Vordergrund. Hinzu kommt, dass in den älteren Listen weitere semiotische Ressourcen der Schriftgestaltung wie eine andere Färbung und Majuskelschreibung genutzt wurden, um gesellschaftliche Ordnungen und sozialen Status darzustellen. Diese Mittel treten in den jüngeren, ergänzten Listen nur noch selten in Erscheinung. Ebenso sind listenimmanente Ordnungen nicht mehr im selben Grad konstruiert oder gar mittels Tilgung manipuliert, sondern hängen vielmehr vom jeweiligen situativen Kontext zum Zeitpunkt der Verschriftung ab - vorausgesetzt, dass aus den Namenlisten überhaupt eine innere Ordnung der zusammengehörigen Personen rekonstruiert werden kann. Die nach inhaltlichen Prinzipien konstruierten älteren Listen stehen somit den situativ und kontextuell zufälligeren jüngeren Listen entgegen.

Mit diesem funktionalen Wandel einhergehend könnte sich auch die Platzierung der Handschrift im jeweiligen Konvent verändert haben, so dass sie für mehrere Schreiber - lokale wie auswärtige - zur Niederschrift zugänglich war. Darunter fanden sich auch weniger geübte Schreiber als dies bei den Anlageschichten der Fall war. Diese waren möglicherweise außerdem weniger vertraut mit anderen mittelalterlichen Textsorten wie beispielsweise Glossaren, in denen Listen vorkamen und damit schreibpraktisch weniger geschult.

Es bleibt jedoch zu erwähnen, dass die schriftliche Verfestigung des eigenen Namens in einem als Liber vitae angelegten Buch an sich eine semiotische Konstante und den Rahmen konstituiert, der der memorialen Funktion überhaupt zu Grunde liegt. Die Niederschrift eines Namens in diesem Buch bedeutete, dass der eigenen Person gedacht wurde. Die eindimensionale Liste stellt laut Algazi (2014, S. 28) überhaupt den Prototyp eines stabilen, interpretativen Rahmens für diesen kulturellen Kontext dar: »You're either in or out, mentioned (that is, >remembered <, but actually $>$ commemorated $<$ ) or omitted ( $>$ forgotten $<$ ) «. Dies kann sowohl religiös aufgefasst werden, d.h. ist man mit Namen in der Liste erfasst, wird die eigene Seele ins Gebet eingeschlossen und man steht nach der biblischen Vorstellung am Jüngsten Tag in der Gunst Gottes, als auch sozial politisch, indem gewisse Personen in ein Kollektiv integriert oder ausgeschlossen werden.

In den Listen der Libri vitae lässt sich die Wandelbarkeit und Flexibilität der Liste als Kulturpraxis und Medium anschaulich aufzeigen. Von Contzen (2017, S. 319f.) spricht im Zusammenhang mit der »Offenheit des Funktionsspektrums bei gleichzeitig relativer Geschlossenheit der Form« von >Affordanzen〈 der Liste, die sich im jeweiligen konkreten Handlungskontext entfalten. Dabei denkt sie den Begriff rein aus der Perspektive der Anwendung und des Gebrauchsnutzens, d.h. in Bezug auf die Rezeption von Listen (von Contzen 2017, S. 324). In den hier behandelten Listen zeigen sich allerdings nicht nur Neukonfigurationen der funktionalen Anwendung der Listen im diachronen Wandel und in unterschiedlichen institutionellen, beziehungsweise politischen Kontexten, sondern auch, dass neue Anwendungs- 
zwecke gar bei praktischen Listen zu modifizierten Formen führen. Der relativ enge Rahmen der sozialen Praxis in mittelalterlichen Libri vitae veranschaulicht somit eine Verschränkung der Rezeptions- und Produktionsseite: Ein veränderter konkreter Handlungskontext führt nicht nur zu neuen Funktionsmöglichkeiten der Listen, sondern auch zu modifizierten Formen, in denen die semiotischen Ressourcen der Schrift in unterschiedlichen Spektren ausgenutzt werden.

Abschließend soll nicht unerwähnt bleiben, dass die interdisziplinäre Zusammenarbeit zwischen Restaurator/inn/en, Philolog/inn/en, Sprachwissenschaftler/ inne/ $n$ und Historiker/inne/ $n$ - wie sich an mehreren Punkten im vorliegenden Beitrag gezeigt hat - für die kulturelle Deutung der Listen in Libri vitae unabdingbar ist.

Open Access Dieser Artikel wird unter der Creative Commons Namensnennung 4.0 International Lizenz (http://creativecommons.org/licenses/by/4.0/deed.de) veröffentlicht, welche die Nutzung, Vervielfältigung, Bearbeitung, Verbreitung und Wiedergabe in jeglichem Medium und Format erlaubt, sofern Sie den/die ursprünglichen Autor(en) und die Quelle ordnungsgemäß nennen, einen Link zur Creative Commons Lizenz beifügen und angeben, ob Änderungen vorgenommen wurden.

\section{Quellen- und Literaturverzeichnis}

\section{Quellen}

Autenrieth, Johanne/Geuenich, Dieter/Schmid, Karl (Hg.) (1979): Das Verbrüderungsbuch der Abtei Reichenau. (MGH. Libri memoriales et Necrologia. N. S. I). Hannover 1979.

Bruckner, Albert/Sennhauser, Hans Rudolf/Perret, Franz (Hg.): Liber Viventium Fabariensis. Basel 1973. Forstner, Karl (Hg.): Das Verbrüderungsbuch von St. Peter in Salzburg. Vollständige Faksimile-Ausgabe im Originalformat der Handschrift Al aus dem Archiv von St. Peter in Salzburg. (Codices Selecti 51). Graz 1974.

Geuenich, Dieter/Ludwig, Uwe (Hg.): Der Memorial- und Liturgiecodex von San Salvatore, Santa Giulia in Brescia. (Monumenta Germaniae Historica. Libri memoriales et necrologia. Nova series 4). Hannover 2000.

Geuenich, Dieter/Ludwig, Uwe: Die St. Galler Verbrüderungsbücher. (Libri momoriales et Necrologia, Nova Series) (in Vorbereitung).

Keynes, Simon: The Liber Vitae of the New Minster and Hyde Abbey, Winchester: British Library Stowe 944, together with leaves from British Library Cotton Vespasian A. VIII and British Library Cotton Titus D. XXVII. (Early English Manuscripts in Facsimile 26). Copenhagen 1996.

Hlawitschka, Eduard/Schmid, Karl/Tellenbach, Gerd: Liber memorialis von Remiremont. (Monumenta Germaniae historica. Libri memoriales 1). Dublin/Zürich 1970.

Rollason, David W./Rollason, Lynda (Hg.): The Durham Liber Vitae London, British Library, MS Cotton Domitian A. VII. Edition and digital facsimile with introduction, codicological, prosopographical and linguistic commentary, and indexes. London 2007.

Rollason, Lynda: The Thorney Liber Vitae (London, British Library, additional MS 40,000, fols I-I2R): edition, facsimile and study. Woodbridge/Suffolk, UK, 2015.

\section{Literatur}

Algazi, Gadi: »Forget Memory: Some Critical Remarks on Memory, Forgetting and History«. In: Sebastian Scholz/Gerald Schwedler/Kai-Michael Sprenger (Hg.): Damnatio in Memoria: Deformation und Gegenkonstruktionen von Geschichte. Wien/Köln/Weimar 2014, S. 25-34.

Autenrieth, Johanne: »Beschreibung des Codex«. In: Johanne Autenrieth/Dieter Geuenich/Karl Schmid (Hg.): Das Verbrüderungsbuch der Abtei Reichenau. Hannover 1979, S. XV-XLI.

Belknap, Robert E.: The list. The uses and pleasures of cataloguing. New Haven 2004.

Contzen, Eva von: »The Limits of Narration: Lists and Literary History«. In: Style 50.3 (2016), S. 241-260. 
Contzen, Eva von: »Die Affordanzen der Liste«. In: Zeitschrift für Literaturwissenschaft und Linguistik 47 (2017), S. 317-326.

Diesenberger, Maximilian: »Das Salzburger Verbrüderungsbuch«. In: Peter Erhart/Jakob Kuratli (Hg.): Bücher des Lebens - Lebendige Bücher. St. Gallen 2010, S. 31-35.

Doležalová, Lucie (Hg.): The charm of a list: From the Sumerians to computerised data processing. Newcastle upon Tyne (2009a).

Doležalová, Lucie: »Ad hoc Lists of Bernard Itier (1163-1225), Librarian of St. Martial de Limoges«. In: Lucie Doležalová (Hg.): The charm of a list: From the Sumerians to computerised data processing. Newcastle upon Tyne (2009c), S. 80-99.

Doležalová, Lucie: »The potentials and limitations of studying lists (Introduction)«. In: Lucie Doležalová (Hg.): The charm of a list: From the Sumerians to computerised data processing. Newcastle upon Tyne (2009b), S. 1-8.

Eco, Umberto: Die unendliche Liste. München 2009.

Erhart, Peter: »Die St. Galler Verbrüderungsbücher im Restaurierungsatelier«. In: Peter Erhart/Jakob Kuratli (Hg.): Bücher des Lebens - Lebendige Bücher. St. Gallen 2010, S. 47-50.

Fludernik, Monica: »Descriptive Lists and List Descriptions«. In: Style 50.3 (2016), S. 309-326.

Geuenich, Dieter: »Die Namen des Verbrüderungsbuchs. Ihre Aufnahme, Lemmatisierung und Wiedergabe in den Registern«. In: Johanne Autenrieth/Dieter Geuenich/Karl Schmid (Hg.): Das Verbrüderungsbuch der Abtei Reichenau. Hannover 1979, S. XLII-LIX.

Geuenich, Dieter: »Das Reichenauer Verbrüderungsbuch«. In: Dieter Geuenich/Uwe Ludwig (Hg.): Libri vitae. Gebetsgedenken in der Gesellschaft des Frühen Mittelalters. Köln 2015, S. 123-146.

Geuenich, Dieter/Ludwig, Uwe: Libri vitae. Gebetsgedenken in der Gesellschaft des Frühen Mittelalters. Köln (2015a).

Geuenich, Dieter/Ludwig, Uwe: »Einleitung«. In: Dieter Geuenich/Uwe Ludwig (Hg.): Libri vitae. Gebetsgedenken in der Gesellschaft des Frühen Mittelalters. Köln (2015b), S. 9-13.

Goody, Jack: The domestication of the savage mind. (Themes in the social sciences). Cambridge 1977.

Insley, John: »The Old English and Scandinavian Personal Names of the Durham Liber Vitae to $1200 \ll$. In: Dieter Geuenich/Uwe Ludwig (Hg.): Libri vitae. Gebetsgedenken in der Gesellschaft des Frühen Mittelalters. Köln 2015, S. 441-452.

Jørgensen, Ellen/Jónsson, Finnur: »Nordiske Pilegrimsnavne i Broderskabsbogen fra Reichenau«. In: Aarbøger for nordisk Oldkyndighed og Historie 13 (1923), S. 1-36.

Karlsson, Susanne: »Multimodalitet i listproduktion«. In: Språk och interaktion 2 (2010), S. 141-170.

Kettemann, Walter: »Ein Namen-Text. Die Churer Bischofsreihe und die politische Botschaft des ältesten Eintrags im >Liber viventium Fabariensis «. In: Peter Erhart/Jakob Kuratli (Hg.): Bücher des Lebens - Lebendige Bücher. St. Gallen 2010, S. 90-95.

Ledin, Per: »Listans och tabellens semiotik «. In: Sakprosa 7.1 (2015), S. 1-25.

Leonardi, Claudio (Hg.): Fabula in tabula: Una storia degli indici dal manoscritto al testo elettronico. Atti del Convegno di Studio della Fondazione Ezio Franceschini e della Fondazione IBM Italia Certosa del Galluzzo, 21-22 ottobre 1994. (Quaderni di cultura mediolatina 13). Spoleto 1995.

Lieven, Jens: »Der >Liber viventium < von Pfäfers. Zum historischen Zeugniswert einer liturgischen Handschrift «. In: Peter Erhart/Jakob Kuratli (Hg.): Bücher des Lebens - Lebendige Bücher. St. Gallen 2010, S. 83-88.

Lieven, Jens: »Großgruppeneinträge in den Libri memoriales. Anmerkungen zu Bischöfen der späten Karolingerzeit im Kontext großer Gruppen«. In: Dieter Geuenich/Uwe Ludwig (Hg.): Libri vitae. Gebetsgedenken in der Gesellschaft des Frühen Mittelalters. Köln 2015, S. 239-272.

Ludwig, Uwe: Transalpine Beziehungen der Karolingerzeit im Spiegel der Memorialüberlieferung prosopographische und sozialgeschichtliche Studien unter besonderer Berücksichtigung des Liber vitae von San Salvatore in Brescia und des Evangeliars von Cividale. (Monumenta Germaniae Historica. Studien und Texte 25). Hannover 1999.

Ludwig, Uwe (Hg.): Nomen et fraternitas. Festschrift für Dieter Geuenich zum 65. Geburtstag. (Ergänzungsbände zum Reallexikon der germanischen Altertumskunde 62). Berlin 2008.

Ludwig, Uwe: »Wann ist das jüngere Verbrüderungsbuch entstanden?«. In: Peter Erhart/Jakob Kuratli (Hg.): Bücher des Lebens - Lebendige Bücher. St. Gallen 2010, S. 51-58.

Ludwig, Uwe: »Die beiden St. Galler Libri vitae aus dem 9. Jahrhundert«. In: Dieter Geuenich/Uwe Ludwig (Hg.): Libri vitae. Gebetsgedenken in der Gesellschaft des Frühen Mittelalters. Köln 2015, S. $147-173$.

Mainberger, Sabine: »Liste und Zeit«. In: Susanne Deicher/Ahmad Elnassan (Hg.): Die Liste. Ordnungen von Dingen und Menschen in Ägypten. Berlin 2015, S. 263-286. 
Naumann, Hans-Peter: »Die altnordischen Personennamen im Verbrüderungsbuch der Abtei Reichenau«. In: Harald Burger/Alois M. Haas/Peter von Matt (Hg.): Verborum Amor. Studien zur Geschichte und Kunst der deutschen Sprache. Festschrift für Stefan Sonderegger zum 65. Geburtstag. Berlin/New York 1992, S. 701-730.

Schaffrick, Matthias/Werber, Niels: »Die Liste, paradigmatisch«. In: Zeitschrift für Literaturwissenschaft und Linguistik 47 (2017), S. 303-316.

Schmid, Karl: »Wege zur Erschließung des Verbrüderungsbuches«. In: Johanne Autenrieth/ Dieter Geuenich/Karl Schmid (Hg.): Das Verbrüderungsbuch der Abtei Reichenau. (MGH. Libri memoriales et Necrologia. Nova series I). Hannover 1979.

Schmid, Karl: »Versuch einer Rekonstruktion der St. Galler Verbrüderungsbücher des 9. Jahrhunderts«. In: Michael Borgolte/Dieter Geuenich/Karl Schmid (Hg.): Subsidia Sangallensia I. Materialien und Untersuchungen zu den Verbrüderungsbüchern und zu den älteren Urkunden des Stiftsarchivs St. Gallen. (St. Galler Kultur und Geschichte 16). St. Gallen 1986, S. 81-283.

Schmid, Karl: »Mönchtum und Verbrüderung«. In: Kottje Raymund/Helmut Maurer (Hg.): Monastische Reformen im 9. und 10. Jahrhundert. (Vorträge und Forschungen 38). Stuttgart 1989, S. 117-146.

Schmid, Karl/Oexle, Otto G.: »Voraussetzungen und Wirkung des Gebetsbundes von Attigny«. In: Francia 2 (1974), S. 71-122.

Stäheli, Urs: »Das Soziale als Liste«. Zur Epistemologie der ANT. In: Friedrich Balke/Maria Muhle/Antonia von Schöning (Hg.): Die Wiederkehr der Dinge. (Kaleidogramme 77). Berlin 2012, S. 83-101.

Wagner, Robert: »Abzuklärende Personennamen aus den Verbrüderungsbüchern von St. Gallen, der Reichenau, Pfäfers und Salzburg«. In: Beiträge zur Namenforschung. Neue Folge 45 (2011), S. 381-450.

Waldispühl, Michelle: »Flerspråkiga pilgrimer? En kontaktonomastisk pilotstudie av de nordiska namnen från Reichenau«. In: Tom Schmidt/Inge Særheim (Hg.): Namn som kjelder. (NORNA-rapporter 92). Uppsala 2017, S. 261-76.

Waldispühl, Michelle: »>Deutschく oder >nordgermanischく? Sprachliche Bestimmung von Namen im Reichenauer Verbrüderungsbuch vor dem Hintergrund von Sprachkontakt und Mehrsprachigkeit«. In: Christoph Hoffarth/Benjamin Scheller (Hg.): Ambiguität und die Ordnungen des Sozialen im Mittelalter. (Das Mittelalter. Perspektiven mediävistischer Forschung. Beihefte 10). Berlin 2018, S. 129-150.

Waldispühl, Michelle: »Historische Rufnamen im Kontakt. Integration der altisländischen Pilgernamen auf der Reichenau in die mittelhochdeutsche Schreibsprache«. In: Luise Kempf/Damaris Nübling/ Mirjam Schmuck (Hg.): Linguistik der Eigennamen. (Linguistik - Impulse und Tendenzen). Berlin (im Druck).

Zettler, Alfons: »>Visio Wettini< und Reichenauer Verbrüderungsbuch«. In: Peter Erhart/Jakob Kuratli (Hg.): Bücher des Lebens - Lebendige Bücher. St. Gallen 2010, S. 59-69. 